Original article

\title{
The effect of HIV infection and exposure on cognitive development in the first two years of life in Malawi
}

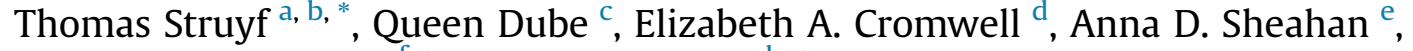 \\ Robert S. Heyderman ${ }^{\mathrm{f}, \mathrm{g}}$, Annelies Van Rie ${ }^{\mathrm{b}, \mathrm{e}}$ \\ a Department of Public Health and Primary Care, KU Leuven, Leuven, Belgium \\ b Department of Epidemiology and Social Sciences, University of Antwerp, Antwerp, Belgium \\ ${ }^{\text {c } Q u e e n ~ E l i z a b e t h ~ C e n t r a l ~ H o s p i t a l, ~ B l a n t y r e, ~ M a l a w i ~}$ \\ ${ }^{\mathrm{d}}$ Institute for Health Metrics and Evaluation, University of Washington, Seattle, USA \\ e Department of Epidemiology, University of North Carolina, Chapel Hill, NC, USA \\ ${ }^{\mathrm{f}}$ Malawi-Liverpool-Wellcome Trust Clinical Research Programme, Blantyre, Malawi \\ g University College London, London, UK
}

\section{A R T I C L E I N F O}

\section{Article history:}

Received 15 October 2018

Received in revised form

30 May 2019

Accepted 17 November 2019

\section{Keywords:}

Cognitive development

HIV

AIDS

Infants

Africa

\begin{abstract}
A B S T R A C T
Objectives: To assess longitudinal patterns and determinants of cognitive development in infants living with HIV, infants exposed to maternal HIV infection, and HIV-unexposed infants.

Methods: Prospective, community-based cohort study of 555 Malawian infants aged 8 weeks to 24 months, using multivariable linear mixed-effects regression models with random intercepts to analyze repeated measures of cognitive function.

Results: At 3 months of age, cognitive scores on the Bayley Scales of Infant Development (BSID 3rd edition) were lower in the 96 HIV-infected infants (mean = 14.1 (SD:4.8)) compared to the 289 HIVexposed (mean $=16.5($ SD:3.7)) and the 170 unexposed infants (mean $=17.5$ (SD:3.3)). Over the first two years of life, the small deficit in cognitive development of infants living with HIV who survived and remained in care did not increase (mean score 52.9 among HIV-infected vs 55.6 among HIV unexposed). In multivariable analysis, malnutrition and a more advanced clinical infant HIV stage had a negative impact on cognition at age 3 , while financial security, care by the biological mother, and ART for mother and child were associated with better cognitive status at this young age. The positive influence of maternal ART reversed with age.

Conclusions: Malawian infants exposed to HIV had a cognitive development that was similar to their unexposed peers in the first two years of life, while that of HIV infected infants lagged behind from the start. Early initiation of effective ART in all HIV infected mothers and infants, and prevention of infant malnutrition are important to safeguard cognitive development of children affected by HIV.
\end{abstract}

๑) 2019 European Paediatric Neurology Society. Published by Elsevier Ltd. All rights reserved.

\section{Introduction}

Every year, there are about 160,000 new Human Immunodeficiency Virus (HIV) infections in children [1]. More than $90 \%$ of these occur in the developing world, and almost all are due to perinatal Mother To Child Transmission (MTCT) or breastfeeding [2]. An important consequence of perinatal HIV infection is the impact on the development of the child's central nervous system (CNS) [3-8].

\footnotetext{
* Corresponding author. Department of Public Health and Primary Care, KU Leuven, Kapucijnenvoer 33 Block J Box 7001, 3000, Leuven, Belgium.

E-mail address: thomas.struyf@kuleuven.be (T. Struyf).
}

The reported prevalence of delay in cognition, motor function, speech and language in perinatally HIV-infected children has varied from low (8\%, high-income countries) to high ( $>60 \%$, sub-Saharan Africa). Prevalence of neurodevelopmental delay increases with age and level of immunodeficiency, and was highest when access to antiretroviral treatment (ART) was still limited [4,7-17]. The highest incidence rate of HIV-related CNS manifestations occurs in the first two years of life, with an estimated incidence of $9.9 \%$ in the first year of life, $4.2 \%$ in the second and less than $1 \%$ thereafter [18-21]. ART can improve neurologic outcomes [6,15,22,23], with treated children showing better recovery towards developmental milestones than their untreated counterparts [24]. 


\begin{tabular}{|ll|}
\hline \multicolumn{2}{|l|}{ Abbreviations } \\
AIC & Akaike Information Criterion \\
AIDS & Acquired Immune Deficiency Syndrome \\
ART & Antiretroviral Therapy \\
BIC & Bayesian Information Criterion \\
BSID-III & Bayley Scales of Infant and toddler Development, \\
& 3rd edition \\
CNS & Central Nervous System \\
DNA & Deoxyribonucleic Acid \\
LOCF & Last Observation Carried Forward \\
MTCT & Mother to Child Transmission (of HIV) \\
NNRTI & Non-Nucleoside Reverse Transcriptase Inhibitor \\
NRTI & Nucleoside Reverse Transcriptase Inhibitor \\
PCR & Polymerase Chain Reaction \\
PMTCT & Prevention of Mother to Child Transmission (of \\
& HIV) \\
RNA & Ribonucleic Acid \\
SD & Standard Deviation \\
WHO & World Health Organization \\
-2LL & Deviance Statistic \\
\end{tabular}

In 2008, the CHER study demonstrated that very early ART, initiated before 12 weeks of age, improves clinical and immunological outcomes compared to ART initiated after immunodeficiency develops [25]. Emerging evidence suggests there may be a benefit to cognitive development of very early ART initiation [25,26].

Apart from HIV infection itself, psychosocial and contextual factors such as poverty, limited family resources, malnutrition and stressful child rearing environments, impairment in overall functioning and increased hospitalization may all further impact the cognitive development of these infants [27-32].

Even though more than $80 \%$ of young infants (age 0-2) living with HIV reside in sub-Saharan Africa, little is known about their cognitive development and its predictors, especially in the context of access to early infant diagnosis (using a DNA PCR assay) and early ART initiation (test and treat strategy) [33]. To date, studies have focussed on older children and found that significant cognitive deficits were present among children at school age living with HIV, even when started on ART at an 'early' age (median $=1.2$ years) [15,34].

We performed a longitudinal, prospective, observational study of perinatal HIV-infected infants, HIV-exposed uninfected infants, and HIV unexposed infants in Blantyre, Malawi, to describe their longitudinal patterns of cognitive development and to identify predictors of their cognitive development.

\section{Methods}

\subsection{Study setting and participants}

A total of 555 infants, of which 96 perinatally HIV-infected, 289 HIV-exposed and 170 non-exposed, were enrolled at two health clinics in Blantyre, Malawi, from January 2008 till June 2011. Pregnant women living with and without HIV were identified through the Department of Health PMTCT program in Blantyre (funded by UNICEF), which provided HIV counseling and testing of all pregnant women and nevirapine for PMTCT to women living with HIV and their newborns. Other PMTCT regimens and universal treatment were not yet available during the study period [2]. All infants born to mothers living with HIV were offered participation in an early infant HIV testing program at age 6 weeks, provided by the study as this was not yet standard of care. All infants living with HIV were referred for care and treatment to the closest pediatric ART center [35].

Infants born to a mother living with HIV were classified as HIV infected if infection was demonstrated by a positive HIV DNA PCR test on dried blood spot and confirmed by a second HIV DNA or a quantitative HIV RNA viral load test. Infants born to a mother living with HIV were classified as HIV exposed uninfected if the absence of HIV infection was demonstrated by a negative PCR test for HIV at enrollment and all follow-up visits. Infants were considered HIV unexposed if born to a mother with documented HIV negative status at the time of antenatal visit and the infant tested negative on a rapid HIV antibody test performed after age 18 months. All HIV-infected infants and all HIV-exposed uninfected infants were invited for study participation. To ensure recruitment of a random sample of HIV unexposed infants over a similar recruitment period, we invited one in 8 HIV exposed infants.

\subsection{Data collection}

At enrollment, clinical and anthropometric data on the infant, and socioeconomic and health status of the mother/caregiver was collected on a standardized form. Socioeconomic status was estimated by inquiring about food security, financial security, housing and education level of the caregiver. Presence of malnutrition and stunting was interpreted according to the WHO definitions [36]. An infant was considered malnourished or stunted when a weight for age Z-score was below 2 SD of the reference mean.

The Bayley Scales of Infant Development, 3rd edition, (BSID-III) were used to assess the cognitive development of all children at age 10-14 weeks, and at months $6,9,12,15,18$ and 24 . These scales have been validated for the use in Malawian children [37]. All tests were administered by study staff (medical officers, pediatricians and nurses) trained in the use of the BSID-III. Because the scoring system employed by the Bayley results in a rate of change over age that is not necessarily constant, raw test scores were used in the analysis to assess the developmental trajectories and the influence of potential predictors on those trajectories.

\subsection{Data analysis}

To analyze the repeated measures of cognitive function during the first two years of life, analyses were conducted using a multivariable linear mixed-effects regression model with random intercepts (PROC MIXED procedure, SAS 9.3), fitted by maximumlikelihood methods [38]. The analysis included all infants with one or more assessments of cognitive development and coped with missing values via correct specification of the likelihood function. We assumed that data was missing at random, i.e. the probability of missingness of data, conditional on all observed outcomes, was unrelated to unobserved concurrent outcomes. Missing outcomes ( $10 \%$ of all outcome data) were imputed using a multiple imputation technique. An iterative algorithm was used for full maximum likelihood estimation of the fixed and random portions of the models.

The functional, linear form of the models was checked at both levels, and visual inspection of the residual distributions revealed the tenability of the normality assumptions. The homoscedasticity assumption was checked by plotting raw residuals against predictors at the respective levels of the models.

Three models were developed by stepwise inclusion of $a$ priori determined covariates. A covariate was retained in the model if it had a significant effect on the initial cognitive score or cognitive growth rate, or if the fit statistics (Deviance, AIC, BIC and $\mathrm{R}^{2}$ ) of the 
model improved after inclusion of the variable, in order to increase the precision of the fixed effects estimates. Time-varying predictors were not retained because the model fit did not improve when including them into the random effects of the models. An unstructured error covariance structure fitted the data best.

HIV-exposed infants who seroconverted during follow-up ( $n=21 / 289$ ), were censored from the analysis on the date the first HIV-positive DNA PCR test was collected.

Sample sizes for the HIV-infected, HIV-exposed and unexposed groups were determined a priori using data from a pilot study in the Democratic Republic of the Congo [4]. Assuming a 14\% mother-tochild transmission rate of HIV by age 6 weeks, a vertical transmission rate of $5 \%$ between age 6 weeks and 6 months, loss of follow up rates of $15 \%$ among HIV unexposed infants, $10 \%$ among HIV exposed uninfected infants and 5\% among HIV infected infants, the intended sample size to achieve $80 \%$ power was $130 \mathrm{HIV}$ infected, 150 HIV exposed uninfected, and 150 unexposed infants.

All statistical analyses were performed using SAS software, Version 9.3 (SAS Institute Inc., Cary, NC, USA).

\subsection{Ethics approval}

The study was approved by the University Of Malawi College Of Medicine Research Ethics Committee, the University of North Carolina Institutional Review Board and the University of Antwerp Ethics Committee. Parents or guardians of all participants provided written informed consent before study enrollment.

\section{Results}

\subsection{Study population}

A total of 555 infants (96 HIV-infected, 289 HIV-exposed uninfected and 170 HIV unexposed) were enrolled at a median age of 11.5 weeks (range $8-78$ weeks) (Table 1 ). Half (51\%) of the infants was female, $13.9 \%$ were malnourished and $43.5 \%$ stunted during follow-up. Malnutrition and stunting were more frequent in infants living with HIV (22.3\% and 50.4\%) and HIV-exposed uninfected infants (15.2\% and $46.0 \%$ ) compared to HIV-unexposed infants $(8.5 \%$ and $36.5 \%)$. Most infants (96.4\%) were cared for by their biological mother. The educational status of the mothers was low, with $48.6 \%$ of the mothers living with HIV and $36.1 \%$ of HIV negative mothers not attending formal education beyond primary school. Socioeconomic status of the infants' households was poor, independent of HIV exposure/infection status. Overall, $25.1 \%$ of caregivers reported financial insecurity, $91.7 \%$ did not have access to a flush toilet, and $99 \%$ lived in a house with a grass (16.5\%) or iron sheet roof (82.5\%). During the two-year follow-up period, hospitalization rate was low (3\%), even among HIV infected children (4.6\%). HIVinfected and HIV-exposed uninfected children were more likely to die (21.9\% and 9.3\% resp.) compared to and HIV unexposed children (3.5\%). HIV-exposed and unexposed children died at an older age (median age at death resp. 36 and 34 weeks) than HIVinfected children (median age at death 18.5 weeks).

\subsection{Cognitive development in the first two years of life}

Cognitive scores at 15 weeks of age were significantly lower in HIV-infected infants (mean $=14.1$ ) compared to HIV-exposed (mean $=16.5)$ and unexposed infants (mean $=17.5)$, but the difference was lower than the 5 difference points which is considered clinically relevant. Furthermore, population variability in cognitive scores within each group was moderate (SD of 4.8, 3.7, and 3.3 for HIV infected, HIV exposed uninfected and HIV unexposed groups, respectively), resulting in differences between the groups that were not statistically significant from age 10 months onwards (Table 2, Fig. 1).

Fig. 1 depicts the mean trajectories of cognitive development in the first two years of life for the three groups. The small deficit in cognitive development did not increase among children living with HIV who survived and remained in care until age 2 . The trajectories of an HIV-exposed uninfected child and an HIV unexposed child were similar from age 15 weeks to age 2 years.

\subsection{Factors associated with impaired cognitive development}

The cognitive scores of infants living with HIV at age 3 months were negatively associated with infant malnutrition (Table 3 ). The difference in population average initial cognitive score between malnourished and non-malnourished HIV-infected infants was -3.48 (95\%CI -5.12 to -1.84$)$, meaning that, on average, a malnourished child would have a 3.48 points lower cognitive score than a child who is not suffering from malnutrition at age 3 months, if all other variables in the model are kept the same. A more advanced clinical HIV stage of a child also had a negative effect on cognition (on average -1.16 points per stage (95\%CI: 1.95 to -0.37$)$ ).

Initial cognitive development scores of infants living with HIV were positively associated with maternal ART $(+3.48,95 \% \mathrm{CI}: 0.59$ to 6.38), but this effect reversed into a negative influence over time ( -7.10 annually, 95\%CI: 11.08 to -3.12 ). A 3-month old HIV-infected infant whose mother is on ART at that time would thus have a 3.48 point higher cognitive score compared to an infant whose mother is not on ART, but on average, over the first two years of life, would lose 7.10 points per year in comparison with an infant whose mother is not on ART, if all other variables in the model would have the same value in both infants. Initial cognitive development scores of infants living with HIV were also positively associated with ART in the infant $(+2.76,95 \% \mathrm{CI}: 0.41$ to 5.12$)$ and with financial security $(+1.15,95 \% \mathrm{CI}: 0.50$ to 1.79$)$. There was no association between maternal ART and infant cognitive development in HIV-exposed uninfected infants.

At age 3 months, care by the biological mother was positively associated with cognitive development scores in infants living with HIV $(+4.17,95 \%$ CI: 0.99 to 7.34$)$, HIV-exposed uninfected $(+9.70$, 95\%CI: 7.15 to 12.25 ) and unexposed infants ( $+5.83,95 \% \mathrm{CI}$ : 3.40 to 8.25). This effect declined with age among HIV-exposed uninfected $(-11.08,95 \% \mathrm{CI}$ : 14.66 to -7.50$)$ and HIV unexposed infants $(-8.66$, $95 \% \mathrm{CI}$ : 14.20 to -3.11 ). Stunting had a negative effect over time in the HIV-exposed uninfected ( $-2.28,95 \% \mathrm{CI}$ : 3.19 to -1.36$)$ and HIV unexposed infants $(-2.57,95 \% \mathrm{CI}: 3.63$ to -1.50$)$.

Note that the magnitude of the effect estimates cannot be compared across groups, because of the fact that they are conditional on all the other variables in the models.

\section{Discussion}

In this prospective cohort study, Malawian infants living with HIV diagnosed through an early infant diagnosis program and referred for early ART initiation had a small, clinically insignificant, cognitive deficit, with a 2.4 point lower mean cognitive score on the BSID-III at age 3 months compared to HIV-exposed uninfected and a 3.4 lower BSID-III score compared to HIV-unexposed infants. This small, but biologically relevant deficit (more than 1 SD below the mean of the HIV-unexposed infants) [39] did not increase in the first 2 years of life among the children who survived and remained in HIV care. We also did not observe a difference in cognitive development between HIV exposed uninfected and HIV unexposed infants at age 3 months nor during the first two years of life. 
Table 1

Characteristics of 555 children, stratified by HIV infection and exposure status (N, \% unless noted).

\begin{tabular}{|c|c|c|c|c|}
\hline Characteristic & HIV-Infected $(\mathrm{N}=96)$ & HIV- Exposed $(\mathrm{N}=289)$ & HIV unexposed $(\mathrm{N}=170)$ & $\begin{array}{l}\text { Total } \\
(\mathrm{N}=555)\end{array}$ \\
\hline Age at enrollment (weeks, median $+\mathrm{IQR}^{\mathrm{a}}$ ) & $13.7(4.7)$ & $12.0(4.1)$ & $10.8(2.0)$ & $11.6(4.0)$ \\
\hline \multicolumn{5}{|l|}{ Gender } \\
\hline Male & 37 (39\%) & $144(50 \%)$ & $89(52 \%)$ & $271(49 \%)$ \\
\hline Female & $59(61 \%)$ & $145(50 \%)$ & $81(48 \%)$ & $285(51 \%)$ \\
\hline $\begin{array}{l}\text { Malnutrition }\left(\mathrm{WAZ}^{\mathrm{b}}<-2 \mathrm{SD}\right) \text { during } 2 \text { year follow-up } \\
\quad(\text { mean } \%+95 \% \text { Confidence Interval })\end{array}$ & $22.3 \%(18.1 \%-26.5 \%)$ & $15.2 \%(13.5 \%-16.9 \%)$ & $8.5 \%(6.7 \%-10.2 \%)$ & $13.9 \%(12.7 \%-15.2 \%)$ \\
\hline $\begin{array}{l}\text { Stunting }\left(\mathrm{HAZ}^{\mathrm{C}}<-2 \mathrm{SD}\right) \text { during } 2 \text { year follow-up } \\
\quad(\text { mean } \%+95 \% \text { Confidence Interval) }\end{array}$ & $50.4 \%(45.3 \%-55.5 \%)$ & $46.0 \%(43.6 \%-48.4 \%)$ & $36.5 \%(33.4 \%-39.5 \%)$ & $43.5 \%(41.7 \%-45.3 \%)$ \\
\hline Mortality during 2 year follow-up & $21(21.9 \%)$ & $27(9.3 \%)$ & $6(3.5 \%)$ & $54(9.7 \%)$ \\
\hline Age at death (weeks, median $+\mathrm{IQR}^{\mathrm{a}}$ ) & $18(11)$ & $36(38)$ & $34(14)$ & $27.5(25)$ \\
\hline \multicolumn{5}{|l|}{ Caregiver relationship } \\
\hline Biological mother & $88(91.7 \%)$ & $280(96.9 \%)$ & $164(96.5 \%)$ & $532(95.8 \%)$ \\
\hline Other & $8(8.3 \%)$ & $9(3.1 \%)$ & $6(3.5 \%)$ & $23(4.2 \%)$ \\
\hline \multicolumn{5}{|l|}{ Socioeconomic characteristics } \\
\hline \multicolumn{5}{|l|}{ Financial insecurity } \\
\hline None of the time & $8(9.5 \%)$ & $33(12.7 \%)$ & $12(8.5 \%)$ & $53(10.9 \%)$ \\
\hline Some of the time & $39(46.9 \%)$ & $123(47.3 \%)$ & $80(56.7 \%)$ & $242(49.8 \%)$ \\
\hline Most of the time & $8(9.5 \%)$ & $41(15.8 \%)$ & $20(14.2 \%)$ & $69(14.2 \%)$ \\
\hline All of the time & $29(34.5 \%)$ & $63(24.2 \%)$ & $29(20.6 \%)$ & $121(25.1 \%)$ \\
\hline \multicolumn{5}{|l|}{ Food insecurity } \\
\hline All of the time & $5(5.9 \%)$ & $9(3.4 \%)$ & $2(1.4 \%)$ & $16(3.3 \%)$ \\
\hline Most of the time & $4(4.8 \%)$ & $11(4.2 \%)$ & $9(6.4 \%)$ & $24(4.9 \%)$ \\
\hline Some of the time & $23(27.4 \%)$ & $86(32.7 \%)$ & $43(30.5 \%)$ & $152(31.1 \%)$ \\
\hline None of the time & $52(61.9 \%)$ & $157(59.7 \%)$ & $87(61.7 \%)$ & $296(60.7 \%)$ \\
\hline \multicolumn{5}{|l|}{ Material of the roof } \\
\hline Grass/other & $15(17.9 \%)$ & $37(14.1 \%)$ & $29(20.3 \%)$ & $81(16.5 \%)$ \\
\hline Iron sheets & $68(80.9 \%)$ & $222(84.4 \%)$ & $114(79.7 \%)$ & $404(82.5 \%)$ \\
\hline Tiles & $1(1.2 \%)$ & $4(1.5 \%)$ & $0(0.0 \%)$ & $5(1.0 \%)$ \\
\hline \multicolumn{5}{|l|}{ Toilet facilities } \\
\hline No toilet & $0(0.0 \%)$ & $1(0.4 \%)$ & $0(0.0 \%)$ & $1(0.2 \%)$ \\
\hline Pit or bucket & $82(97.6 \%)$ & $236(89.1 \%)$ & $132(92.3 \%)$ & $451(91.5 \%)$ \\
\hline Outdoor flush toilet & $0(0.0 \%)$ & $4(1.5 \%)$ & $0(0.0 \%)$ & $4(0.8 \%)$ \\
\hline Indoor flush toilet & $2(2.4 \%)$ & $24(9.1 \%)$ & $11(7.7 \%)$ & $37(7.5 \%)$ \\
\hline \multicolumn{5}{|l|}{ Maternal education level } \\
\hline None & $8(9.5 \%)$ & $24(9.0 \%)$ & $4(2.8 \%)$ & $6(7.3 \%)$ \\
\hline Some primary school & $31(36.9 \%)$ & $89(33.5 \%)$ & $39(27.1 \%)$ & $160(32.3 \%)$ \\
\hline Completed primary school & $12(14.3 \%)$ & $23(8.6 \%)$ & $9(6.2 \%)$ & $44(8.9 \%)$ \\
\hline Some secondary school & $19(22.6 \%)$ & $64(24.1 \%)$ & $60(41.7 \%)$ & $143(28.9 \%)$ \\
\hline Completed secondary school or higher education & $14(16.7 \%)$ & $66(24.8 \%)$ & $32(22.2 \%)$ & $112(22.6 \%)$ \\
\hline Number of siblings living with the child (mean $+\mathrm{SD}$ ) & $1.69(1.20)$ & $1.93(1.43)$ & $1.64(1.42)$ & $1.82(1.42)$ \\
\hline Nevirapine in infant (PMTCT) & $71(74.1 \%)$ & $261(90.3 \%)$ & NA & NA \\
\hline ART in mother & $16(18.8 \%)$ & $83(29.5 \%)$ & NA & NA \\
\hline \multicolumn{5}{|l|}{ ART in infant } \\
\hline at 15 weeks & $4(4.2 \%)$ & NA & NA & NA \\
\hline at 2 years & $24(92.3 \%)$ & NA & NA & NA \\
\hline
\end{tabular}

a $\mathrm{IQR}=$ Inter Quartile Range (75th percentile- 25th percentile).

b $\mathrm{WAZ}=$ Weight for Age Z-score $(\mathrm{WHO})$.

${ }^{c} \mathrm{HAZ}=$ Height for Age Z-score $(\mathrm{WHO}) ; \mathrm{SD}=$ Standard deviation.

d Financial insecurity = Answering the question: "In the past week, how often did you have enough money to meet your needs?"

e Food insecurity = Answering the question: "In the past week, how often were you hungry because you did not have enough food?"

Table 2

Mean cognitive levels (raw Bayley scores) and 95\% confidence intervals at 15 weeks and at 2 years, by subgroup.

\begin{tabular}{|c|c|c|c|c|}
\hline & & HIV-infected children $(\mathrm{N}=96)$ & HIV-exposed children $(\mathrm{N}=289)$ & Control children $(\mathrm{N}=170)$ \\
\hline \multirow[t]{2}{*}{ Cognitive score at 15 weeks } & Mean $\left(\mathrm{SD}^{\mathrm{a}}\right)$ & $14.11(4.8)$ & $16.50(3.7)$ & $17.49(3.3)$ \\
\hline & $95 \%$ confidence interval & $12.92-15.30$ & $16.04-16.97$ & $16.94-18.04$ \\
\hline \multirow[t]{2}{*}{ Cognitive score at 2 years } & Mean $\left(\mathrm{SD}^{\mathrm{a}}\right)$ & $52.91(6.9)$ & $55.61(6.8)$ & $54.78(7.4)$ \\
\hline & $95 \%$ confidence interval & $50.47-55.34$ & $54.56-56.66$ & $53.30-56.25$ \\
\hline
\end{tabular}

${ }^{\mathrm{a}} \mathrm{SD}=$ Standard Deviation.

Studies on development of HIV infected infants on ART in resource poor settings at a very young age are scarce. While several studies have shown that infants reached their developmental gross and fine motor outcome milestones at a later age than their healthy peers $[24,40]$, cognitive outcomes at this young age have not yet been reported. Studies in infants and children have shown a developmental "catch up", but mainly when ART was initiated in older symptomatic children who were not yet severely immunosuppressed [14,41]. Older children may also perform better as a result of a "survival effect," as those infants with less aggressive disease are more likely to survive [4].

In our cohort of Malawian infants living with HIV, maternal ART was associated with better cognitive development at a very young age. We were unable to identify other studies assessing the effect of 


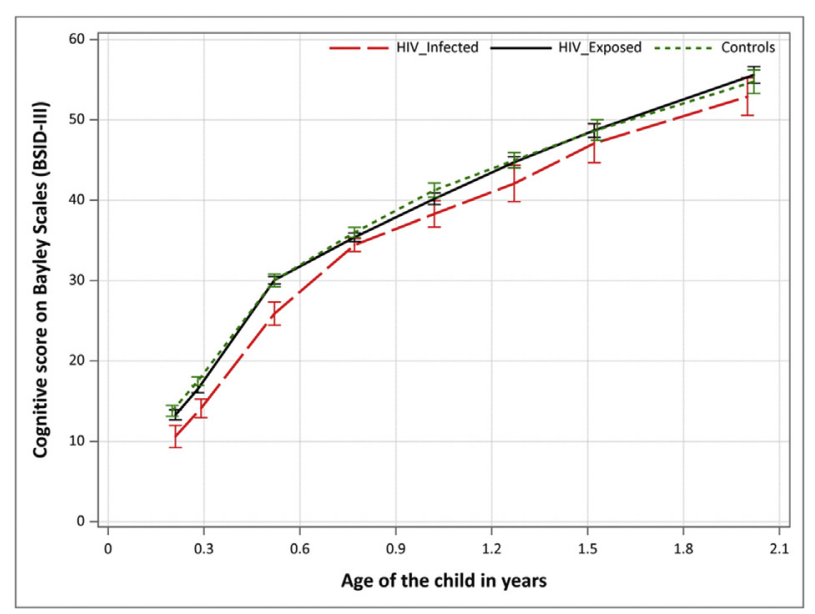

Fig. 1. Mean cognitive trajectories of HIV-infected, HIV-Exposed and unexposed children, including $95 \%$ confidence intervals. (Use of color in printed version is not necessary).

maternal ART on the cognitive status of perinatally infected infants. We hypothesize this effect is due to an absolute reduction in the perinatal exposure of the infant to the virus, as maternal ART lowers the viral load during pregnancy and the perinatal period. This finding, as well as the finding that HIV-exposed but uninfected infants perform similar to unexposed infants, strengthen the call for universal option B + for PMTCT, test-and-treat for all women living with HIV, and safer conception programs to prevent horizontal HIV transmission during the earliest stages of pregnancy [42-50].

In contrast to the positive effect at age 3 months, the effect of maternal ART reversed with age. While this finding may be counter-intuitive, we believe it could, at least in part, be explained by the fact that in our cohort mothers were only eligible for ART when they had developed severe immunodeficiency (CD4 count $<250$ cells $/ \mathrm{mm} 3$ ) [51]. The impaired health status of mothers on ART compared to that of mothers not yet eligible for ART could result in decreased capability of parenting $[52,53]$. This hypothesis is supported by the fact that care by the biological mother was another factor positively associated with cognitive development at a very young age in infants living with HIV. Similarly, the positive effect of care by the biological mother may be explained by the fact that mothers living with HIV who were able to care for and nurture their infants themselves were healthier compared to those mothers who could not take on the role of primary caregiver. This effect should disappear in a setting where universal ART is implemented.

Malnutrition negatively impacted cognitive development in infants living with HIV at age 3 months. Malnutrition has been associated with both structural and functional pathology of the brain in HIV uninfected infants, which can lead to a wide range of cognitive deficits, especially during the first months of life $[54,55]$.

In our cohort, early initiation of infant ART did benefit the initial cognitive level but not afterwards. Because of the observational study design, the absence of an effect of ART on cognitive development in our cohort cannot be interpreted as the absence of a causal effect of ART on cognitive development. Even though all infants were to be started on ART 'upon diagnosis', the infrastructure for early infant diagnosis of HIV, with transport of dried blood spots for HIV DNA PCR at a centralized laboratory and the requirement to confirm a possible infant HIV infection resulted in a delay in ART initiation, with an average age of ART initiation of 30.3 weeks and $19.8 \%$ of infants dying prior to initiating any ART. In the sickest children, ART was initiated prior to confirmation of HIV status, which may have resulted in confounding by indication.

Furthermore, at the time of the study, nevirapine was the only

Table 3

Results of fitting the linear mixed-effects models to the data, by subgroup.

\begin{tabular}{|c|c|c|c|c|}
\hline & & Model A: HIV-Infected $n=96$ & Model B: HIV-Exposed $n=289$ & Model C: Controls $\mathrm{n}=170$ \\
\hline \multicolumn{5}{|l|}{ Fixed effects } \\
\hline \multirow[t]{10}{*}{ Initial Status (at 3 months) } & Intercept & $13.57^{* *}(8.8015 .52)$ & $12.16^{* * *}(10.6119 .52)$ & $17.98^{* * *}(15.64$ 20.32) \\
\hline & Caregiver $=$ mother & $4.16^{*}(0.997 .34)$ & $9.70^{* * *}(7.1512 .25)$ & $5.83^{* * *}(3.408 .25)$ \\
\hline & ART(child) & $2.76^{*}(0.415 .12)$ & & \\
\hline & ART(mother) & $3.48^{*}(0.596 .38)$ & & \\
\hline & PMTCT (infant) & $0.76(-0.772 .29)$ & $0.20(-0.971 .38)$ & \\
\hline & Clinical stage child & $-1.16^{* *}(-1.95-0.37)$ & & \\
\hline & Malnutrition & $-3.48^{* * *}(-5.12-1.84)$ & $-0.61(-1.600 .37)$ & $-0.55(-2.090 .99)$ \\
\hline & Financial insecurity & $1.15^{* *}(0.501 .79)$ & $-0.04(-0.420 .34)$ & \\
\hline & Number of siblings & & $0.12(-0.130 .37)$ & \\
\hline & Material roof & & $0.65(-0.281 .57)$ & \\
\hline \multirow[t]{6}{*}{ Rate of change } & Intercept (Time) & $25.21^{* * *}(21.2729 .16)$ & $32.77^{* * *}(28.4837 .07)$ & $29.93^{* * *}(24.3935 .47)$ \\
\hline & ART (mother)*TIME & $-7.10^{* *}(-11.08-3.12)$ & no sign. effect ${ }^{\mathrm{a}}$ & \\
\hline & ART (infant)*TIME & no sign. effect ${ }^{\mathrm{a}}$ & & \\
\hline & Stunting*TIME & & $-2.28^{* * *}(-3.19-1.36)$ & $-2.57^{* * *}(-3.63-1.50)$ \\
\hline & caregiver $=$ mother*TIME & & $-11.08^{* * *}(-14.66-7.50)$ & $-8.65^{* *}(-14.20-3.11)$ \\
\hline & Toilet facilities*TIME & & $0.23(-0.651 .11)$ & \\
\hline \multicolumn{5}{|l|}{ Variance components } \\
\hline Residual variance $\left(\sigma_{\varepsilon}^{2}\right)$ & & $26.30^{* * *}$ & $27.75^{* * *}$ & $28.85^{* * *}$ \\
\hline Initial status $\left(\sigma_{0}^{2}\right)$ & & 0 & 0 & 0 \\
\hline Covariance $\left(\sigma_{01}\right)$ & & $-8.23^{* *}$ & -1.49 & -0.42 \\
\hline Rate of change $\left(\sigma^{2}{ }_{1}\right)$ & & $23.14^{* *}$ & $6.49^{* *}$ & $7.17^{*}$ \\
\hline \multicolumn{5}{|l|}{ Fit statistics } \\
\hline$-2 L L^{b}$ & & 1455.9 & 6523.4 & 4821.1 \\
\hline AIC & & 1483.9 & 6551.4 & 4839.1 \\
\hline BIC & & 1515.4 & 6599.0 & 4866.5 \\
\hline Adjusted $\mathrm{R}^{2}$ & & 0.80 & 0.81 & 0.78 \\
\hline
\end{tabular}

${ }^{*} \mathrm{p}<.05 ;{ }^{* *} \mathrm{p}<.01 ;{ }^{* * *} \mathrm{p}<.00195 \%$ Confidence Intervals in brackets.

Note: SAS Proc Mixed, Full ML

a Predictors ART(infant)*Time and ART(Mother)*Time were removed from the model, because they did not improve model fit.

b $-2 \mathrm{LL}=$ Deviance statistic. 
Table 4

Resistance rates to NRTI's and to NNRTI's after 1 year of life in HIV-infected children.

\begin{tabular}{|c|c|c|}
\hline & Resistance to NNRTI's n/N, (\%) & Resistance to NRTI's n/N, (\%) \\
\hline Tested samples from HIV-infected children after 1 year of life $(n=36)$ & $23 / 36(63.9 \%)$ & $12 / 36(33.3 \%)$ \\
\hline
\end{tabular}

NNRTI: Non-Nucleoside Reverse Transcriptase Inhibitor.

NRTI: Nucleoside Reverse Transcriptase Inhibitor.

drug available for prevention of mother to child transmission, and the combination of nevirapine, lamivudine and stavudine was the only available antiretroviral drug regimen, initially as split adult tablets and later as pediatric syrup. Under these conditions, resistance to the prescribed ART's (NNRTI + NRTI) in our cohort was high, with $63.9 \%$ of infants tested after 1 year of life $(n=36)$ having documented mutations associated with resistance of the virus (Table 4). Consequently, while children were on ART, many may not have received effective ART and thus not have been virally suppressed. The importance of viral suppression on neurodevelopment was demonstrated in one study, where young HIV-infected infants with viral suppression on ART were found to have better motor development better than those without suppression on ART [24].

The only factors associated with cognitive development of HIVexposed and unexposed infants were stunting, which was detrimental to cognitive development in the first two years of life, and care by the biological mother, which was beneficial for the cognitive status at age 3 months but negatively associated with cognitive development after age 3 months. This is in contrast to other studies in older infants and children in the US, which have found that care by the mother, including maternal education level, is important for cognitive development, in combination with the availability of sufficient household resources and an adequate level of nutrition $[33,56,57]$.

Important strengths of the study include the longitudinal cohort design with careful assessment of HIV status at very young age and throughout the first two years of life, the multiple measurements of cognitive development using a validated and widely used BSID instrument, and the inclusion of both HIV exposed and HIV unexposed infants from the same communities as controls. Some limitations should however be noted when interpreting the results. First, due to a well-functioning prevention of mother to child program, only 96 out of the intended 130 perinatally infected infants were recruited. Furthermore, due to a combination of loss-to follow-up and a high level of mortality, the sample size of infants living with HIV was relatively small, especially after the first year of life. The use of a linear mixed-effects regression model however allowed us to use all available data, even if an infant only contributed a single visit. A disadvantage of this type of analysis is the requirement to use the raw Bayley scores that change systematically over time. Because this method was not used in other studies, this limited our ability to compare results with prior studies. Second, given the continued improvements made in the global policies regarding prevention of mother to child transmission and infant ART, our findings may underestimate what can be achieved under current program conditions.

A final limitation is the fact that HIV prevention and treatment policies have changed since the observations included in the analysis. We believe that our findings are still important and relevant. First, even though our sample of children living with HIV was smaller than intended, it is still the largest study of neurodevelopment of infants age $0-2$ years living with HIV. Second, the observation that we could not find important differences in cognitive development under suboptimal circumstances suggests that it is highly unlikely these would be observed under current, vastly improved PMTCT and ART programs. Third, the observation that the cognitive development of HIV exposed uninfected children is not different from HIV unexposed infants is important as this is a growing and highly vulnerable population in sub-Saharan Africa which is likely to persist for decades to come [42].

\section{Conclusion}

In the context of early infant diagnosis and early initiation of ART, infants living with HIV had a small but biologically relevant cognitive deficit at age 3 months. This deficit did not increase among infants who survived and remained in HIV care in the first two years of life. Infants exposed to HIV who remained uninfected had similar cognition in the first two years of life as HIV unexposed children. Furthermore, the higher mortality observed in infants living with HIV also strengthens the call for effective ART in all pregnant women, safer conception care for all HIV affected couples, very early ART for infants living with HIV, and prevention of malnutrition and stunting.

\section{Funding source}

Funding was provided by the United States National Institutes of Health/National Institute of Child Health and Human Development, via grant R01 HD053216. Malawi-Liverpool-Wellcome is funded through a core program grant from the Wellcome Trust, UK.

\section{Financial disclosure}

The authors have no financial relationships relevant to this article to disclose.

\section{Contributors' statement page}

Mr. Struyf carried out the statistical analyses and drafted the manuscript.

Prof. Van Rie conceptualized and designed the cohort study, supervised data collection, data management and data analysis, and reviewed and revised the manuscript.

Dr. Dube supervised all field activities, assisted in data management, and reviewed and revised the manuscript.

Dr. Cromwell assisted in data management and data analysis, and reviewed and revised the manuscript.

Dr. Sheahan designed data collection instruments, assisted in supervision of field activities, supervised data management, carried out analyses, and reviewed and revised the manuscript.

Prof. Heyderman coordinated and supervised data collection, and critically reviewed the manuscript.

All authors approved the final manuscript as submitted and agree to be accountable for all aspects of the work.

\section{Declaration of competing interest}

The authors have no conflicts of interest relevant to this article to disclose. 


\section{Acknowledgements}

Funding was provided by the United States National Institutes of Health/National Institute of Child Health and Human Development, via grant R01 HD053216. Malawi-Liverpool-Wellcome is funded through a core program grant from the Wellcome Trust, UK. We also thank the participants and their families for their participation.

\section{References}

[1] WHO, Global Summary of the HIV/AIDS Epidemic, December 2016, WHO, Geneva, 2016.

[2] WHO, Antiretroviral Therapy for HIV Infection in Infants and Children: towards Universal Access. Recommendations for a Public Health Approach, WHO, Geneva, 2010.

[3] N. Baillieu, J. Potterton, The extent of delay of language, motor, and cognitive development in HIV-positive infants, J. Neurol. Phys. Ther. : J. Neurol. Phys. Ther. 32 (2008) 118-121.

[4] A. Van Rie, A. Mupuala, A. Dow, Impact of the HIV/AIDS epidemic on the neurodevelopment of preschool-aged children in Kinshasa, Democratic Republic of the Congo, Pediatrics 122 (2008) e123-e128.

[5] B. Laughton, M. Cornell, M. Boivin, A. Van Rie, Neurodevelopment in perinatally HIV-infected children: a concern for adolescence, J. Int. AIDS Soc. 16 (2013) 18603.

[6] C.S. Crowell, K.M. Malee, R. Yogev, W.J. Muller, Neurologic disease in HIVinfected children and the impact of combination antiretroviral therapy, Rev. Med. Virol. 24 (2014) 316-331.

[7] D. The Lancet Infectious, The challenge of HIV associated neurocognitive disorder, The Lancet. Infectious diseases 13 (2013) 907.

[8] D.B. Clifford, B.M. Ances, HIV-associated neurocognitive disorder, Lancet. Infect. Dis. 13 (2013) 976-986.

[9] A. Llorente, P. Brouwers, M. Charurat, et al., Early neurodevelopmental markers predictive of mortality in infants infected with HIV-1, Dev. Med. Child Neurol. 45 (2003) 76-84.

[10] C.L. Gay, F.D. Armstrong, D. Cohen, et al., The effects of HIV on cognitive and motor development in children born to HIV-seropositive women with no reported drug use: birth to 24 months, Pediatrics 96 (1995) 1078-1082.

[11] C. Chase, M. Vibbert, S.I. Pelton, D.L. Coulter, H. Cabral, Early neurodevelopmental growth in children with vertically transmitted human immunodeficiency virus infection, Arch. Pediatr. Adolesc. Med. 149 (1995) $850-855$.

[12] H. Pollack, A. Kuchuk, L. Cowan, et al., Neurodevelopment, growth, and viral load in HIV-infected infants, Brain Behav. Immun. 10 (1996) 298-312.

[13] W.G. Knight, C.A. Mellins, R.L. Levenson Jr., S.M. Arpadi, R. Kairam, Brief report: effects of pediatric HIV infection on mental and psychomotor development, J. Pediatr. Psychol. 25 (2000) 583-587.

[14] A. Van Rie, A. Dow, A. Mupuala, P. Stewart, Neurodevelopmental trajectory of HIV-infected children accessing care in Kinshasa, Democratic Republic of Congo, J. Acquir. Immune Defic. Syndr. 52 (2009) 636-642.

[15] H. Brahmbhatt, M. Boivin, V. Ssempijja, et al., Neurodevelopmental benefits of antiretroviral therapy in Ugandan children aged 0-6 years with HIV, J. Acquir. Immune Defic. Syndr. 67 (2014) 316-322.

[16] A. Van Rie, P.R. Harrington, A. Dow, K. Robertson, Neurologic and neurodevelopmental manifestations of pediatric HIV/AIDS: a global perspective, Eur. J. Paediatr. Neurol. : EJPN : Off. J. Eur. Paediatr. Neurol. Soc. 11 (2007) 1-9.

[17] S.L. Nichols, M.C. Chernoff, K.M. Malee, et al., Executive functioning in children and adolescents with perinatal HIV infection and perinatal HIV exposure, J. Pediatr. Infect. Dis. Soc. 5 (2016) S15-S23.

[18] L.G. Epstein, L.R. Sharer, J.M. Oleske, et al., Neurologic manifestations of human immunodeficiency virus infection in children, Pediatrics 78 (1986) 678-687.

[19] M.N. Lobato, M.B. Caldwell, P. Ng, M.J. Oxtoby, Encephalopathy in children with perinatally acquired human immunodeficiency virus infection. Pediatric Spectrum of Disease Clinical Consortium, J. Pediatr. 126 (1995) 710-715.

[20] M. Tardieu, J. Le Chenadec, A. Persoz, L. Meyer, S. Blanche, M.J. Mayaux, HIV-1 related encephalopathy in infants compared with children and adults. French Pediatric HIV Infection Study and the SEROCO Group, Neurology 54 (2000) 1089-1095.

[21] T.T. Tahan, I. Bruck, M. Burger, C.R. Cruz, Neurological profile and neurodevelopment of 88 children infected with HIV and 84 seroreverter children followed from 1995 to 2002, Braz. J. Infect. Dis. : Off. Publ. Braz. Soc. Infect. Dis 10 (2006) 322-326.

[22] W.J. Muller, Treatment of perinatal viral infections to improve neurologic outcomes, Pediatr. Res. 81 (2017) 162-169.

[23] N. Whitehead, J. Potterton, A. Coovadia, The neurodevelopment of HIVinfected infants on HAART compared to HIV-exposed but uninfected infants, AIDS Care 26 (2014) 497-504.

[24] S. Benki-Nugent, D. Wamalwa, A. Langat, et al., Comparison of developmental milestone attainment in early treated HIV-infected infants versus HIV unexposed infants: a prospective cohort study, BMC Pediatr. 17 (2017) 24

[25] M.F. Cotton, A. Violari, K. Otwombe, et al., Early time-limited antiretroviral therapy versus deferred therapy in South African infants infected with HIV results from the children with HIV early antiretroviral (CHER) randomised trial, Lancet (London, England) 382 (2013) 1555-1563.

[26] B. Laughton, M. Cornell, D. Grove, et al., Early antiretroviral therapy improves neurodevelopmental outcomes in infants, Aids 26 (2012) 1685-1690.

[27] D.A. Hackman, R. Gallop, G.W. Evans, M.J. Farah, Socioeconomic status and executive function: developmental trajectories and mediation, Dev. Sci. 18 (2015) 686-702.

[28] A. Ursache, K.G. Noble, Socioeconomic status, white matter, and executive function in children, Brain Behav. 6 (2016) e00531.

[29] B.S. McEwen, P.J. Gianaros, Central role of the brain in stress and adaptation: links to socioeconomic status, health, and disease, Ann. N. Y. Acad. Sci. 1186 (2010) 190-222.

[30] K.D. Hermetet-Lindsay, K.F. Correia, P.L. Williams, et al., Contributions of disease severity, psychosocial factors, and cognition to behavioral functioning in US youth perinatally exposed to HIV, AIDS Behav. 21 (9) (2017 Sep) $2703-2715$.

[31] M. Mutumba, J.A. Bauermeister, K.S. Elkington, et al., A prospective longitudinal study of mental health symptoms among perinatally HIV-infected and HIV-exposed but uninfected urban youths, J. Adolesc. Health : Off. Publ. Soc. Adolesc. Med. 58 (2016) 460-466.

[32] C.J. Hochhauser, S. Gaur, R. Marone, M. Lewis, The impact of environmental risk factors on HIV-associated cognitive decline in children, AIDS Care 20 (2008) 692-699.

[33] K. Le Doare, R. Bland, M.L. Newell, Neurodevelopment in children born to HIVinfected mothers by infection and treatment status, Pediatrics 130 (2012) e1326-e1344.

[34] M.J. Boivin, L. Barlow-Mosha, M.C. Chernoff, et al., Neuropsychological performance in African children with HIV enrolled in a multisite antiretroviral clinical trial, AIDS 32 (2) (2018) 189-204.

[35] WHO, Antiretroviral Therapy of HIV Infection in Infants and Children: Recommendations for a Public Health Approach, 2006, 2006 revision.

[36] WHO, Global Database on Child Growth and Malnutrition-The Z-Score or Standard Deviation Classification System, 2017.

[37] E.A. Cromwell, Q Dube, S.R. Cole, et al, Validity of US norms for the Bayley scales of infant development-III in Malawian children, Eur. J. Paediatr. Neurol. EJPN : Off. J. Eur. Paediatr. Neurol. Soc. 18 (2014) 223-230.

[38] J.D. Singer, J.B. Willett, Applied Longitudinal Data Analysis- Modeling Change and Event Occurrence, Oxford University Press, 2003.

[39] N. Bayley, Bayley Scales of Infant and Toddler Development-Third Edition: Technical Manual, Harcourt Assessment, San Antonio, TX, 2006b.

[40] B. Laughton, M. Cornell, M. Kidd, et al., Five year neurodevelopment outcomes of perinatally HIV-infected children on early limited or deferred continuous antiretroviral therapy, J. Int. AIDS Soc. 21 (2018) e25106.

[41] L.A. Gomez, C.S. Crowell, I. Njuguna, et al., Improved neurodevelopment after initiation of antiretroviral therapy in human immunodeficiency virus-infected children, Pediatr. Infect. Dis. J. 37 (2018) 916-922.

[42] L.-G. Bekker, V. Black, L. Myer, et al., Guideline on Safer Conception in Fertile HIV-Infected Individuals and Couples, vol 12, 2011, p. 14.

[43] N.E. Davies, L.T. Matthews, T.L. Crankshaw, D. Cooper, S.R. Schwartz, Supporting HIV prevention and reproductive goals in an HIV-endemic setting: taking safer conception services from policy to practice in South Africa, J. Int. AIDS Soc. 20 (2017) 21271

[44] S.E. Forhan, S. Modi, J.C. Houston, L.N. Broyles, Moving toward test and start: learning from the experience of universal antiretroviral therapy programs for HIV-infected pregnant/breastfeeding women, Aids 31 (2017) 1489-1493.

[45] M. Hosseinipour, J.A.E. Nelson, C. Trapence, et al., Viral suppression and HIV drug resistance at 6 Months among women in Malawi's option B+ program: results from the PURE Malawi study, J. Acquir. Immune Defic. Syndr. 75 (Suppl 2) (2017) S149-S155.

[46] S.M. le Roux, K.A. Donald, M. Kroon, et al., HIV viremia during pregnancy and neurodevelopment of HIV-exposed uninfected children in the context of universal antiretroviral therapy and breastfeeding: a prospective study, Pediatr. Infect. Dis. J. 38 (1) (2019 Jan) 70-75.

[47] S.M. le Roux, K.A. Donald, K. Brittain, et al., Neurodevelopment of breastfed HIV-exposed uninfected and HIV-unexposed children in South Africa, Aids 32 (2018) 1781-1791.

[48] P.E. Springer, A.L. Slogrove, B. Laughton, et al., Neurodevelopmental outcome of HIV-exposed but uninfected infants in the mother and infants health study, Cape Town, South Africa, Trop. Med. \& Int. Health 23 (2018) 69-78.

[49] S. Chaudhury, P.L. Williams, G.K. Mayondi, et al., Neurodevelopment of HIVexposed and HIV-unexposed uninfected children at 24 months, Pediatrics (2017) 140.

[50] M.S. McHenry, C.I. McAteer, E. Oyungu, et al., Neurodevelopment in young children born to HIV-infected mothers: a meta-analysis, Pediatrics 141 (2018).

[51] $\mathrm{MOH}$, Treatment of AIDS: Guidelines for the Use of Antiretroviral Therapy in Malawi, third ed., Ministry of Health, Lilongwe, 2008.

[52] IAS, IAS Conference on HIV pathogenesis, treatment and prevention 7th IAS Conference, Kuala Lumpur (2013).

[53] I. Familiar, S.M. Collins, A. Sikorskii, et al., Quality of caregiving is positively associated with neurodevelopment during the first year of life among HIVexposed uninfected children in Uganda, J. Acquir. Immune Defic. Syndr. 77 (2018) 235-242.

[54] B.R. Kar, S.L. Rao, B.A. Chandramouli, Cognitive development in children with chronic protein energy malnutrition, Behav. Brain Funct. : BBF 4 (2008), 31-31.

[55] MAL-ED Ni, The MAL-ED study: a multinational and multidisciplinary 
approach to understand the relationship between enteric pathogens, malnutrition, gut physiology, physical growth, cognitive development, and immune responses in infants and children up to 2 years of age in resourcepoor environments, Clin. Infect. Dis.: Off. Publ. Infect. Dis. Soc. Am. 59 (Suppl 4) (2014) S193-S206.

[56] P.A. Sirois, Y. Huo, P.L. Williams, et al., Safety of perinatal exposure to antiretroviral medications: developmental outcomes in infants, Pediatr. Infect. Dis. J. 32 (2013) 648-655.

[57] C. Chase, J. Ware, J. Hittelman, et al., Early cognitive and motor development among infants born to women infected with human immunodeficiency virus. Women and Infants Transmission Study Group, Pediatrics 106 (2000). E25E25.

Please cite this article as: T. Struyf et al., The effect of HIV infection and exposure on cognitive development in the first two years of life in Malawi, European Journal of Paediatric Neurology, https://doi.org/10.1016/j.ejpn.2019.11.004 